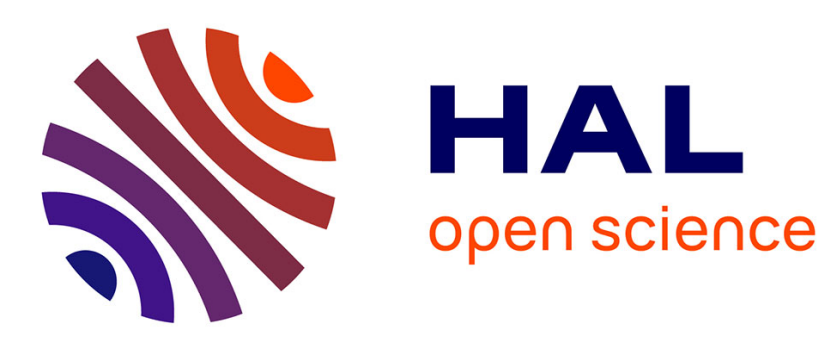

\title{
Observation of liquid-to-crystal transition in a two dimensional electronic system
}

D. Marty, J. Poitrenaud, F.I.B. Williams

\section{To cite this version:}

D. Marty, J. Poitrenaud, F.I.B. Williams. Observation of liquid-to-crystal transition in a two dimensional electronic system. Journal de Physique Lettres, 1980, 41 (13), pp.311-314. 10.1051/jphyslet:019800041013031100 . jpa-00231787

\section{HAL Id: jpa-00231787 https://hal.science/jpa-00231787}

Submitted on 1 Jan 1980

HAL is a multi-disciplinary open access archive for the deposit and dissemination of scientific research documents, whether they are published or not. The documents may come from teaching and research institutions in France or abroad, or from public or private research centers.
L'archive ouverte pluridisciplinaire HAL, est destinée au dépôt et à la diffusion de documents scientifiques de niveau recherche, publiés ou non, émanant des établissements d'enseignement et de recherche français ou étrangers, des laboratoires publics ou privés. 


\title{
Observation of liquid-to-crystal transition in a two dimensional electronic system
}

\author{
D. Marty, J. Poitrenaud and F. I. B. Williams \\ DPh-G/PSRM, CEN Saclay, B.P. No 2, 91190 Gif sur Yvette, France
}

(Reçu le 13 mars 1980, accepté le 9 mai 1980)

\begin{abstract}
Résumé. - En étudiant le signal d'absorption des électrons à deux dimensions déposés à la surface de l'hélium liquide et excités par un champ électrique de fréquence fixe $25 \mathrm{MHz}$, nous avons observé la transition liquidecristal.
\end{abstract}

\begin{abstract}
We have observed the liquid-to-crystal transition in a two dimensional system of electrons at the surface of liquid helium, by studying the absorption signal of the electrons excited by an electric field of a fixed frequency $25 \mathrm{MHz}$.
\end{abstract}

Electrons constrained to move on a flat surface constitute an excellent system on which to test ideas on two dimensional ordering transitions. The interaction is exactly known and is characterized by a single parameter, the charge $e$, for the ideal case of unrestricted geometry. In the classical limit one can then construct two characteristic energies $e^{2} n^{1 / 2}$ for the Coulomb interaction and $k T$ for the temperature ( $n$ is a real number density). Then the relevant variable is

$$
\Gamma=\pi^{1 / 2} e^{2} n^{1 / 2} / k T .
$$

One expects order as $\Gamma \rightarrow \infty$, disorder as $\Gamma \rightarrow 0$. Infinitely long range spatial order in two dimensions only exists for $\Gamma=\infty$, but if one applies instead the criterion for a solid of Kosterlitz and Thouless [1] of long range topological order (closure of Burgers circuits), one finds a liquid-to-solid transition for $\Gamma_{\mathrm{c}} \approx 80$ [2]. Recent computer estimates based on comparison of free energies of liquid and solid configurations give $\Gamma=125 \pm 15$ [3].

The experiment described here is a measure of the melting temperature $T_{\mathrm{c}}$ of a system of electrons condensed onto a liquid helium surface which constitutes a good approach, to such a two dimensional system. We observe the variation of signal due to longitudinal vibrations (or plasmons) with the temperature.

The liquid surface although very smooth is deformable and this facilitates the detection of the transition. Shikin [4] pointed out that an electron held onto a liquid surface depresses the liquid beneath it. The binding energy of a single electron to its deformation is typically of the order of a few $\mathrm{mK}$ under our conditions. When $N$ particles are rigidly correlated the binding energy of the ensemble $(N \times$ a few $\mathrm{mK})$ is to be compared with the thermal energy $T$ of its centre of mass motion. In the liquid state where correlation extends over only a few particles the substrate deformation is not bound to the particles for our temperatures $(>0.3 \mathrm{~K})$ and the dynamics can be treated independently predicting a plasmon spectrum [5] :

$$
\omega_{\mathrm{p}}^{2}(k)=\frac{2 \pi n e^{2}}{m} k \frac{2}{\operatorname{cotanh}(k a)+\operatorname{cotanh}(k b)},
$$

where the last factor corrects for non infinite electrical geometry $(k$ is the wave vector of the excitation in the plane of the electrons $z=0$, itself parallel to two plane parallel conductors at $z=a$ and $z=-b$, see Fig. 1). These modes were first observed by Grimes and Adams [6].

In the solid state, however, the correlation quickly extends over hundreds of particles and the substrate deformation becomes bound to the electrons. As pointed out by Fisher et al. [7] the dynamics must then take into account the motion of the correlated surface deformation to which we can ascribe an in-plane displacement variable with an associated dynamical mass $m^{*}$ per unit cell. The physical picture is one of an electron lattice riding on a waffled surface with the 


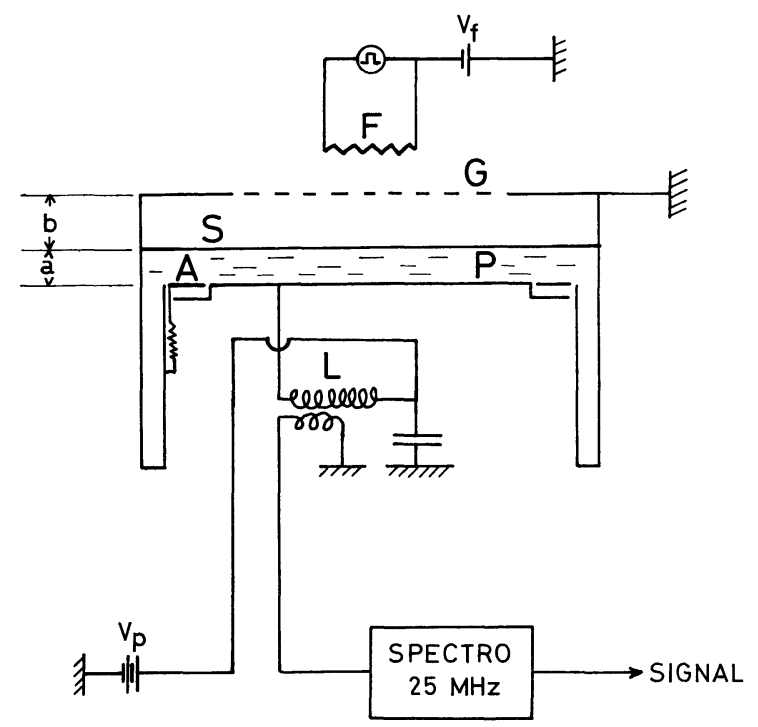

Fig. 1. - Schematic view of the experiment cell. A : guard-ring. F. : tungsten filament. $\mathbf{G}$ : grid. L : coil. P : plate. $\mathrm{S}$ : liquid helium surface.

appearance of coupled motion of electron and waffle displacement. As $k \rightarrow 0$ one finds optical and acoustical modes whose longitudinal frequencies are given by :

$$
\begin{aligned}
\omega_{\mathrm{opt}}^{2} & \sim \omega_{\mathrm{L}}^{2}+\omega_{\mathrm{p}}^{2}(k) \\
\omega_{\mathrm{ac}}^{2} & \sim \frac{m}{m^{*}} \omega_{\mathrm{p}}^{2}(k)
\end{aligned}
$$

where $\omega_{\mathbf{L}}^{2} \sim \frac{m^{*}}{m} \omega_{\mathbf{R}}^{2}$ is the local mode frequency and $m^{*}=\frac{e^{2} E_{\perp}^{2} n^{2}}{2 \alpha^{2} g^{2}} \frac{\rho}{n \alpha g}$.

In the particular simple case where $\frac{m^{*}}{m} \gg 1$, $\omega_{\mathrm{ac}}^{2} \ll \omega_{\mathrm{R}}^{2}$ and taking into account only the first cell of the hexagonal reciprocal lattice. $g$ is the reciprocal lattice vector, $\omega_{\mathrm{R}}^{2}=\frac{\alpha}{\rho} g^{3}$ is the ripplon frequency, $\alpha$ the surface tension and $\rho$ the density of the liquid helium substrate. $E_{\perp}$ is the perpendicular holding field on the electrons and we note that under the saturation conditions where the asymptotic field above the electrons is zero $\varepsilon E_{\perp}=2 \pi n e$ and $\omega_{\mathrm{L}}^{2} \sim n^{3}$, unlike $\omega_{\mathrm{p}}^{2} \sim n$.

While for large $k$ ( $k R \gg 1 ; R$ being the cell radius)

$$
\omega_{\mathrm{opt}}^{2} \sim \omega_{\mathrm{p}}^{2}(k) \text { and } \omega_{\mathrm{ac}}^{2} \sim \omega_{\mathrm{R}}^{2} .
$$

Figure 2 shows the variation of $\omega_{\mathrm{p}}$ and $\omega_{\mathrm{opt}}$ versus electron density.

The detection of the acoustic branch with a swept frequency spectrometer enabled Grimes and Adams [8] to affirm for the first time the existence of the electron solid and to measure the transition temperature.

The experiment described here is more sensitive to the behaviour of the optical mode. It consists in

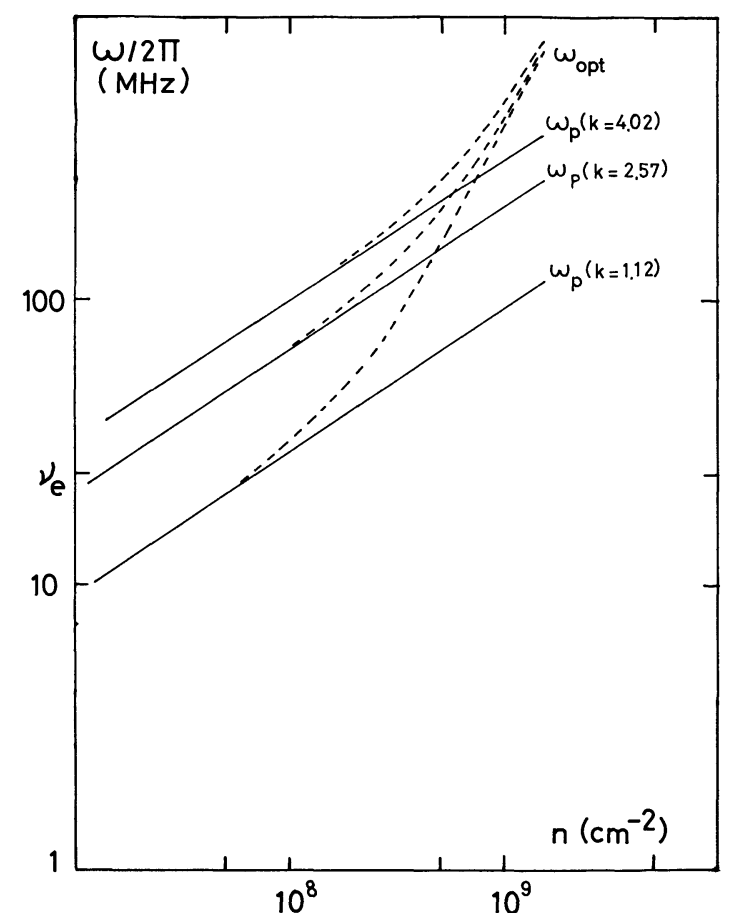

Fig. 2. - Calculated frequency of 2D electronic system longitudinal excitations versus electronic density. Full line : plasmon frequency in the liquid for the three lower modes; wave vector $k$ values are calculated from the limit condition $J_{0}(k R)=0, J_{0}$ being zeroth order Bessel function, $R$ the confinement radius of electrons. Dotted line : electron ripplon coupled modes optical frequency in the solid after [7]; see eq. (2). $v_{\mathrm{e}}$ is the experimental work frequency $(24.5 \mathrm{MHz})$.

monitoring the absorption of energy from in-plane longitudinal component of electric field of fixed wave vectors $\left(k \sim 2 \mathrm{~cm}^{-1}\right.$ and harmonics) and fixed frequency, as a function of temperature and electron density. A sketch of the frequency response of the electrons for fixed $k$ illustrates the situation rather simply, on figure 3 . One readily sees that, to the extent that there exists an absorption at $v_{\mathrm{e}}$ in either phase, the shift of the optical plasmon mode can be detected.

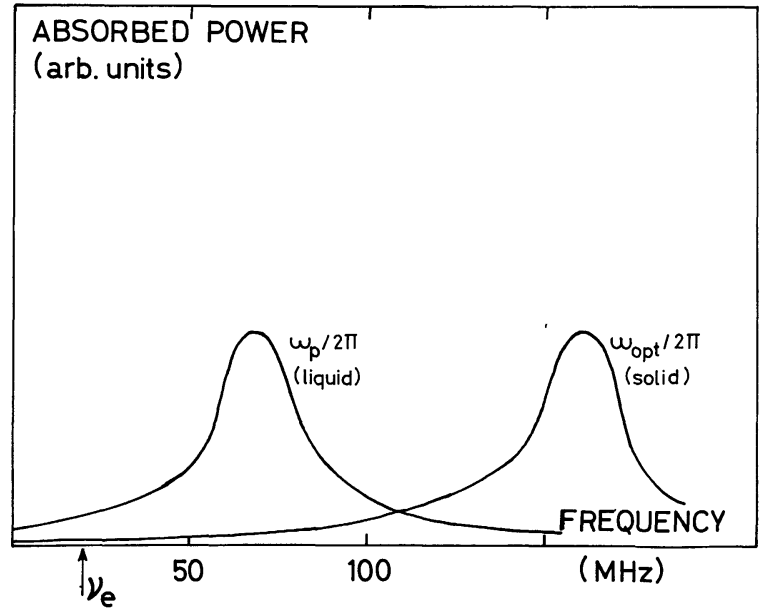

Fig. 3. - Qualitative absorption curves in the 2D liquid (left curve) and in the 2D solid (right curve) for $n=5 \times 10^{8} \mathrm{~cm}^{-2}$ and $k=1.12 \mathrm{~cm}^{-1}$. The damping is presumed the same. 
The acoustic mode contributes little because weak and narrow.

Experimentally we measure the absorption with a fixed frequency $(24.5 \mathrm{MHz})$ heterodyne spectrometer whose quality factor $Q$ is about 1000 at $1.2 \mathrm{~K}$ in the absence of charges.

The free surface of helium lies between the plates of the condenser which together with the coil $\mathrm{L}$ forms the resonant circuit. This high $Q$ circuit was designed to produce principally a vertical component of electric field and to have sufficient sensitivity to detect the generation of ripplons of reciprocal lattice vector for wave vector. The fringe fields however are sufficient for detecting very easily radial electronic modes and in our present conditions these swamp any possible ripplon signal because of the very much lighter effective mass.

Electrons are generated by thermoelectronic emission from a tungsten filament $F$, heated by a $30 \mathrm{~ms}$ pulse. They cross the grid G (see Fig. 1) and settle at the surface $S$, until the electric field due to the surface charge balances the d.c. electric field due to the plate bias, i.e. $\varepsilon V_{\mathrm{p}} / a=4 \pi e n$, where $V_{\mathrm{p}}$ is the voltage between plates, $a$ the lower plate-surface distance, $\varepsilon=1.057$ is the liquid helium dielectric constant and $n$ the electronic density. (One checks $n$ by integrating the excess grid current while discharging the surface.)

Electronic density can be varied up to

$$
0.8 \times 10^{9} \mathrm{~cm}^{-2} \text {. }
$$

The temperature, which may be varied from $0.3 \mathrm{~K}$ to $1.2 \mathrm{~K}$, is established by a helium 3 refrigerator and is measured with carbon resistors, calibrated against ${ }^{3} \mathrm{He}$ vapour pressure. A second ${ }^{3} \mathrm{He}$ refrigerator is used as a thermal anchor to reduce heat leaks, principally that due to a fountain pressure column in the ${ }^{4} \mathrm{He}$ fill capillary.

We have recorded absorption signals versus temperature for different values of voltage $V_{\mathrm{p}}$, i.e. for different values of density $n$. Such a signal is plotted on figure 4. On cooling, the signal increases untill $0.8 \mathrm{~K}$, becomes stationary then rather abruptly decreases from c point, at temperature $T_{\mathrm{c}}$.

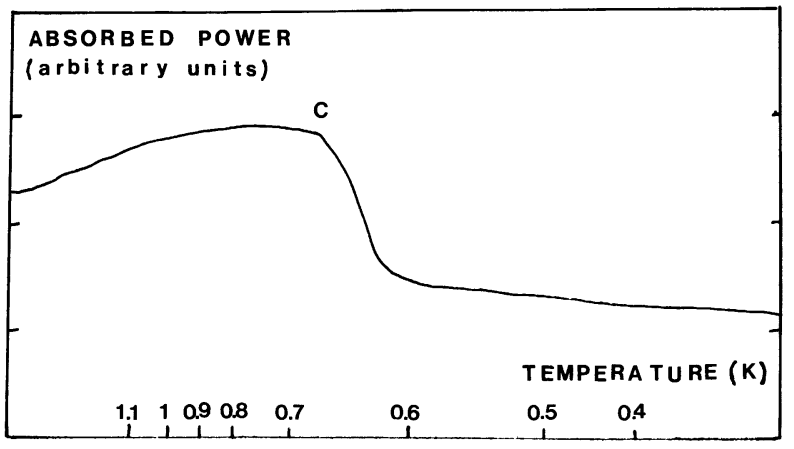

Fig. 4. - Experimental record of the absorption signal versus temperature. The temperature scale is not linear and corresponds to a linear time scanning, during a cooling sequence. Voltage is $V_{\mathrm{p}}=220 \mathrm{~V}$, density $n=6.1 \times 10^{8} \mathrm{~cm}^{-2}$.
For a horizontal excitation field $E_{k} \mathrm{e}^{i \omega t}$, the power absorbed by the electrons is :

$$
A_{k}=\frac{1}{2} \frac{e^{2} E_{k}^{2} S}{m} \frac{n \tau}{1+\omega^{2} \tau^{2}\left[\frac{\omega_{k}^{2}}{\omega^{2}}-1\right]^{2}}
$$

$S$ is the surface occupied by the electrons, $\tau$ the electronic relaxation time (related to mobility by $\mu=e \tau / m), \omega$ the experimental angular frequency $(2 \pi \times 24.5 \mathrm{MHz})$; (at our work frequency $\omega \tau$ varies from 0.9 at $T<0.6 \mathrm{~K}$ to 0.05 at $1.2 \mathrm{~K}) ; \omega_{k}$, the $k$ mode eigenfrequency for plasmons - $\omega_{\mathrm{p}}$ see eq. (1) if in the liquid, and the coupled mode frequency $\omega_{\text {opt }}$ - see eq. (2) - if in the solid.

At temperature $T$ above $T_{\mathrm{c}}$, if we compare our experimental signals with eq. (4) - using mobility values measured by other authors $[6,9]$ - the variation of the signal with temperature qualitatively coincides with the variation of the relaxation time $\tau$. But it is difficult to reach perfect agreement between experiment and theory without knowing the exact Fourier decomposition of the exciting field.

We have interpreted $T_{\mathrm{c}}$ as the liquid-to-crystal transition temperature. One may suppose indeed that, at fixed density, when the temperature is lowered, the signal due to plasmons in the liquid becomes a signal due to the optical mode (of angular frequency $\left.\omega_{\text {opt }}\right)$ in the crystal.

Temperature $T_{\mathrm{c}}$ varies with plate voltage $V_{\mathrm{p}}$ (i.e. with density). We list in table $\mathrm{I}\left(n_{\mathrm{c}}, T_{\mathrm{c}}\right)$ coordinates of these points c. Plotting these points logarithmically, one finds they are rather well aligned on a straight line of slope 1.8 , i.e. $n_{\mathrm{c}} \sim T_{\mathrm{c}}^{1.8}(1.8$ is calculated by the least squares method from experimental points).

In order to compare these results with those of Grimes and Adams [8], we have compared both series on figure 5 , in $\left(n^{1 / 2}, T\right)$ coordinates.

The liquid-to-crystal transition is expected for a constant value of $\Gamma=\pi^{1 / 2} n_{\mathrm{c}}^{1 / 2} e^{2} / k T_{\mathrm{c}}$, i.e. $n_{\mathrm{c}}$ proportional to $T_{\mathrm{c}}^{2}$.

If we calculate $\Gamma$ values from our experimental points (see Table I) we get $\Gamma=118 \pm 10$ (to be

Table I. - c point coordinates and $\Gamma$ values for these points.

$\begin{array}{ccc}n_{\mathrm{c}}\left(10^{8} \mathrm{~cm}^{-2}\right) & T_{\mathrm{c}}(\mathrm{K}) & \Gamma_{\mathrm{c}}=\frac{\pi^{1 / 2} e^{2} n_{\mathrm{c}}^{1 / 2}}{k T_{\mathrm{c}}} \\ - & -\overline{.292} & - \\ 1.44 & 0.295 & 122 \\ 1.48 & 0.302 & 122 \\ 1.60 & 0.315 & 124 \\ 1.64 & 0.357 & 120 \\ 2.04 & 0.395 & 118 \\ 2.48 & 0.435 & 118 \\ 2.92 & 0.476 & 116 \\ 3.34 & 0.59 & 114 \\ 4.95 & & 112\end{array}$




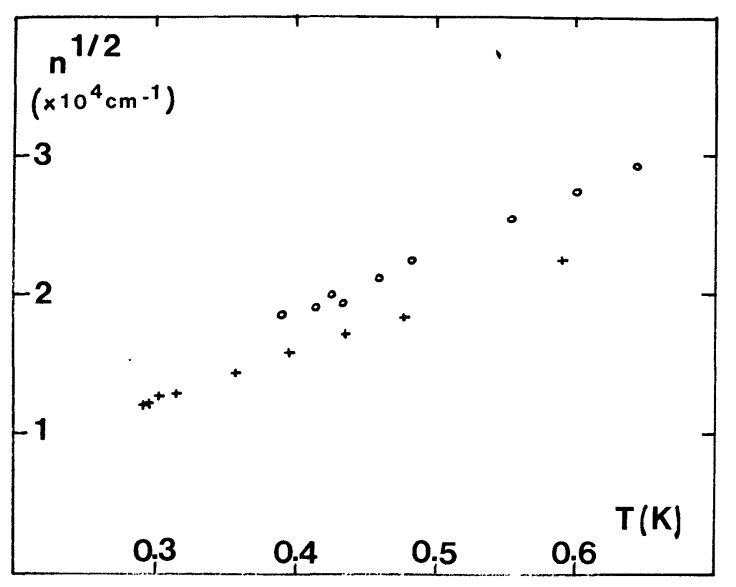

Fig. 5. - Liquid-to-crystal transition in the $\left(n^{1 / 2}, T\right)$ plane. Circles : measurements of Grimes and Adams [8]. Crosses : our measurements (coordinates of « $\mathrm{c} »$ points).

compared with the results of Grimes and Adams [8] : $137 \pm 15$ ). It should be borne in mind when contemplating the discrepancy between these two results that the two experiments do not measure quite the same phenomenon.
Systematic variation of $\Gamma$ with temperature (which corresponds to the fact that we measure a slope 1.8 and not 2, for the straight line $\ln n_{\mathrm{c}}$ versus $\ln T_{\mathrm{c}}$ ) may be due to an imprecision in the determination of $n_{\mathrm{c}}$ (perhaps because of a residual field due to trapped charges).

The measurements we present here result from a slightly different manifestation of the same phenomenon observed by Grimes and Adams [8] and analysed by Fisher et al. [7]. If the method gives very much less and more indirect information on the excitations of the system, it allows one to detect the transition very easily with a very good signal to noise ratio. Our ultimate purpose is to see the ripplon signal with a rf field strictly perpendicular to the surface, but it seemed interesting to describe this result though the discrepancy with the first measurement of $\Gamma$ seems real.

Acknowledgments. - We gratefully acknowledge fruitful discussions with our colleagues, particularly with G. Deville, F. Gallet and A. Valdes. We would also like to pay homage to the technical aid of G. Delmas.

\section{References}

[1] Kosterlitz, J. M. and Thouless, D. J., J. Phys. C 6 (1973) 1181.

[2] Thouless, D. J., J. Phys. C 11 (1978) L 189.

[3] Gann, R. C., Chakravarty, S. and Chester, G. V., Phys. Rev. B 20 (1959) 326.

[4] Shikin, V. B., Pis'ma Zh. Eksp. Teor. Fiz. 68 (1975) 1423; [Sov. Phys. JETP 41 (1976) 710].
[5] Bonsall, L. and Maradudin, A. A., Phys. Rev. B 15 (1977) 1959.

[6] Grimes, C. C. and Adams, G., Phys. Rev. Lett. 36 (1976) 145. [7] Fisher, D. S., Halperin, B. I. and Platzman, P. M., Phys. Rev. Lett. 42 (1979) 798.

[8] Grimes, C. C. and Adams, G., Phys. Rev. Lett. 42 (1979) 795.

[9] Bridges, F. and McGill, J. F., Phys. Rev. B 15 (1977) 1324. 